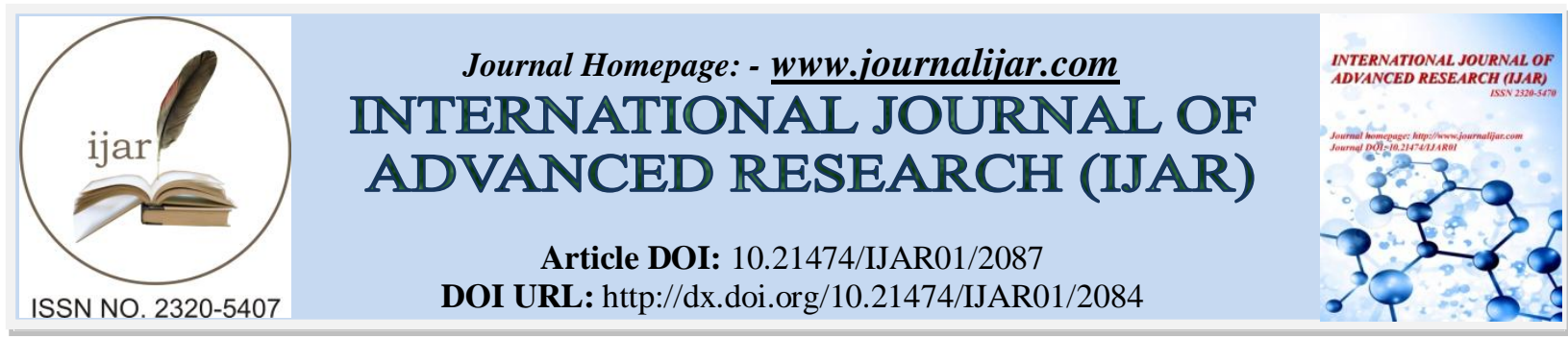

RESEARCH ARTICLE

\title{
MANAGEMENT AND CLINICAL OUTCOME OF SYSTEMIC LUPUS ERYTHEMATOSUS PATIENTS; AT NEPHROLOGY UNIT, ZAGAZIG UNIVERSITY HOSPITALS: A ONE YEAR STUDY.
}

\author{
Amir M. El Okely MD', Adel A.M. Ghorab MD¹, Eman H. Abdelbary MD² and Mahmoud M. Magdy \\ Mahmoud MSc'. \\ 1. Nephrology unit, Internal Medicine Department, Faculty of Medicine, Zagazig University, Egypt. \\ 2. Pathology Department, Faculty of Medicine, Zagazig University, Egypt.
}

\section{Manuscript Info}

\section{Manuscript History}

Received: 24 September 2016

Final Accepted: 26 October 2016

Published: November 2016

Key words:-

SLE, LN, CR, PR, MMF,

cyclophosphamide .

\section{Abstract}

Background: Systemic Lupus Erythematosus (SLE) is a multisystem autoimmune disorder with a broad spectrum of clinical presentations encompassing almost all organs and tissues. Renal disease in SLE carries a significant morbidity and mortality. Despite treatment advances, up to $26 \%$ of patients with lupus Nephritis (LN) still develop End Stage Renal Disease (ESRD).

Objectives: To specify the best lines of management for SLE, those directly influence the outcome of SLE patients in our unit, Also to follow up and compare between the favorable and the unfavorable SLE activity outcomes.

Subjects and methods: Our study was an interventional non randomized clinical trial conducted at Nephrology Unit, Internal Medicine Department, Zagazig University Hospitals, including 64 adult participants diagnosed with SLE who gave their informed consent. All subjects were divided according to their renal biopsy into three groups, the $1^{\text {st }}$ group was 21 patients ( 2 males and 19 females) with class III $(\mathrm{LN})$, the $2^{\text {nd }}$ group was 28 patients ( 3 males and 25 females) with class IV (LN) and the $3{ }^{\text {rd }}$ group was 15 patients ( 2 males and 13 females) with class V-proliferative (LN), each group was subdivided into 2 subgroups according to the induction treatment used that was either oral mycophenolate mofetil (MMF) or iv cyclophosphamide (CYC) .

Results: (LN) class IV is the commonest, proteinuria was higher in class V-proliferative (V+ III/IV) (LN) group than other 2 groups and serum albumin was lower than the other 2 groups. The rates of response have been $31.3 \%$ for complete remission (CR), $45.3 \%$ for partial remission (PR), $20.3 \%$ of patients reported treatment failure, $10.9 \%$ developed ESRD while $17.2 \%$ died .The highest rate of treatment response either CR or PR was noticed with class III (LN), While the highest rate of non responders was noticed with class IV (LN) \& class (V-III/IV) (LN). MMF showed superiority over CYC. 


\section{Introduction:-}

Systemic Lupus Erythematosus (SLE) is a multisystem autoimmune disorder with a broad spectrum of clinical presentations encompassing almost all organs and tissues. SLE is an autoimmune pathology which due to the diversity of its clinical and immunological manifestations represents the prototype of autoimmune connective tissue diseases Lee et al., (2010) (2).

Lupus Nephritis (LN) occurs in up to 60\% of adults with SLE and predicts poor survival. The prevalence of SLE, (LN) and treatment response vary by age, gender, location and race/ethnicity Jakes et al., (2012) (1).

Renal disease represents a frequent manifestation of SLE as well as an important outcome predictor in these patients. As expected, mortality rates are higher in patients with (LN) than in those without renal disease, and some of these (10-60\%) can develop (ESRD) D'Cruz and Houssiau, (2009) (4).

It should be mentioned that (LN) can be resistant to current immunosuppressive regimens. Filling this gap is paramount for the prevention, treatment and cure of (LN). The current clinical management of patients with (LN) remains limited to the use of non-specific cyto-toxic drugs despite the advent of numerous potential biologic agents Kalloo et al., (2013) (5).

Lupus-associated renal damage has been one of the leading causes of 5-year mortality in patients with SLE in the past 50 years. Over the past 5 decades, therapeutic advances, in particular immunosuppression, have significantly improved outcomes for patients with (LN). However, recent evidence indicates that improvement in (LN) outcomes plateaued in the 2000s, perhaps because we have reached the limitations of our current therapies Hoover and Costenbader (2016) (3).

The aim of our study is to determine \& compare management protocols for SLE patients, to better understand the current state of (LN) and to study the clinical outcome of these patients.

\section{Subjects and Methods:-}

This study was an interventional non randomized clinical trial that has been carried out at Nephrology unit, Internal Medicine Department, Zagazig University Hospitals, during the period from (March 2014 to March 2016).

\section{Subjects:-}

Adult patients with (LN) who all were admitted to Nephrology unit and are meeting at least 4 items of SLE diagnostic criteria of the American college of Rheumatology( ACR) 1997 which was updated by the Systemic Lupus International Collaborating Clinics classification criteria (2012 SLICC SLE Criteria). The study included 64 cases with SLE.

All subjects were divided into three groups according to their renal biopsy:

\section{Group 1:-}

This group included 21 patients with Class III (LN), (2 males and 19 females) with age ranging from 18-45 years old with Mean \pm SD $27.42 \pm 8.46$. This group was subdivided -according to their induction treatment- into 2 sub-groups: Group 1A: This group received induction treatment with iv Cyclophosphamide, $(0.5-1 \mathrm{~g} / \mathrm{m} 2)$ given monthly for 6 months (The "NIH regimen"). This group included 12 patients (2 males and 10 females).

Group 1B: This group received oral MMF (maximum $3 \mathrm{~g} / \mathrm{d}$ ) for 6 months. This group included 9 patients (all were females).

\section{Group 2:-}

This group included 28 patients with Class IV (LN), ( 3 males and 25 females) with age ranging from 18-41 years old with Mean \pm SD $27.03 \pm 7.14$. This group was subdivided -according to their induction treatment- into 2 subgroups:

Group 2A: This group received induction treatment with iv Cyclophosphamide, $(0.5-1 \mathrm{~g} / \mathrm{m} 2)$ given monthly for 6 months (The "NIH regimen"). This group included 18 patients ( 2 males and 16 females).

Group 2B: This group received oral MMF (maximum $3 \mathrm{~g} / \mathrm{d}$ ) for 6 months. This group included 10 patients ( 1 male $\& 9$ females). 


\section{Group 3:}

This group included 15 patients with class (V/proliferative) (V-III/IV) (LN), ( 2 males and 13 females) with age ranging from $18-45$ years old with Mean \pm SD $27.86 \pm 6.42$. This group was subdivided -according to their induction treatment- into 2 sub-groups:

Group 3A: This group received induction treatment with iv Cyclophosphamide, $(0.5-1 \mathrm{~g} / \mathrm{m} 2)$ given monthly for 6 months (The "NIH regimen"). This group included 10 patients (1 males and 9 females).

Group 3B: This group received oral MMF (maximum $3 \mathrm{~g} / \mathrm{d}$ ) for 6 months. This group included 5 patients ( 1 male \& 4 females).

In both regimens corticosteroid was used as follow: As an initial dose of iv methylprednisolone (0.5-1 gm/day) for 3 days at the beginning of treatment followed by oral prednisone up to $1 \mathrm{mg} / \mathrm{kg}$, tapering according to clinical response over 6-12 months.

All patients with (LN) of any class are treated with hydroxychloroquine (maximum daily dose of $6-6.5 \mathrm{mg} / \mathrm{kg}$ ideal body weight), unless they have a specific contraindication to this drug Ruiz-Irastorza et al., (2010) (30).

Azathioprine or MMF were used as maintenance therapy after induction and clinical outcomes were compared among different classes (Complete remission, Partial remission, Deterioration or Non- response and ESRD).

Patients were selected according to the following criteria:

\section{Inclusion criteria:-}

1. Adult patients with (LN) who all were admitted at Nephrology unit.

2. Age more than 18 years.

3. Both sexes.

4. Patients consent to share in the study.

\section{Exclusion criteria:-}

1. Patients refusing to share in the study.

2. Age less than 18 years. (Juvenile SLE)

3. Patient refusing renal biopsy.

4. Overlap syndrome.

5. Results of biopsies not consistent with any classes of (LN).

\section{Methods of Study:-}

All subjects of the study were subjected to the following:-

1. Thorough history and full clinical examination.

2. Blood samples were collected for :

a. Routine investigations including:

i. Complete blood picture.

ii. ESR, CRP

iii. Fasting blood glucose.

iv. Lipid profile.

v. Liver function tests.

vi. Pelvi-abdominal ultrasound.

vii. Serum calcium.

viii. Serum creatinine (S.Cr) and BUN.

ix. Serum phosphorus.

x. TSH, free T4, freeT3.

b. Special investigations including:
i. ANA.
ii. Anti- dsDNA.
iii. $\mathrm{C} 3 \& \mathrm{C} 4$.
iv. HCVAb , HBsAg.
v. 24 hours urinary protein.
vi. Renal biopsy. 
3. Assessment of the disease activity by SLEDAI-2K scores. Definitions of response to therapy in (LN) according to KDIGO guidelines:-

Complete response: Return of S.Cr to previous baseline, plus a decline in the 24 hours urinary protein to less than $500 \mathrm{mg} /$ day.

Partial response: Stabilization, or improvement of S.Cr, but not to normal, plus a more than $50 \%$ decrease in 24 hours urinary protein. If there was nephrotic-range proteinuria ( 24 hours urinary protein more than $3 \mathrm{~g} /$ day), improvement requires a more than 50\% reduction in 24 hours urinary protein and a 24 hours urinary protein less than $3 \mathrm{~g} /$ day.

Deterioration: There is no definition of deterioration in $(\mathrm{LN})$ to define treatment failure that has been tested prospectively as an indication to change in initial therapy. A sustained $25 \%$ increase in $\mathrm{SCr}$ is widely used but has not been validated. (KDIGO 2012) (29)

\section{Statistical analysis:-}

All data were collected, tabulated and statistically analyzed using SPSS 20.0 for windows (SPSS Inc., Chicago, IL, USA), MedCalc 13 for windows (MedCalc Software bvba, Ostend, Belgium) and Microsoft Office Excel 2010 for windows (Microsoft Cor., Redmond, WA, USA). Quantitative data were expressed as the mean \pm SD \& median (range), and qualitative data were expressed as absolute frequencies "number"\& relative frequencies (percentage). Continuous data were checked for normality by using the Shapiro Walk test. The independent Student t-test was used to compare two groups of normally distributed data. The Mann-Whitney U was used to compare two groups of non normally distributed data. The One way ANOVA test was used to compare more than two groups of normally distributed data. The Kruskal Wallis $\mathrm{H}$ test was used to compare more than two groups of non normally distributed data. The Post hoc test for multiple comparisons was done by using Tamhane's T2 method. The percentages of categorical variables were compared using the Chi-square test or Fisher's exact test when appropriate. Spearman's coefficient was calculated to assess relationship between study parameters, a (+) sign indicate a direct correlation \& a (-) sign indicates an inverse correlation, while values near to 1 indicate a strong correlation and values near 0 indicate a weak correlation. To determine predictors for subclinical hypothyroidism, univariate logistic regression was done. The backward multivariate logistic regression analysis model was done using any predictor with $p<0.2$ in the univariate analysis. All tests were two sided. $\mathrm{p}<0.05$ was considered statistically significant $(\mathrm{S}), \mathrm{p}<0.001$ was considered highly statistically significant (HS), and $\mathrm{p} \geq 0.05$ was considered non statistically significant (NS).

\section{Results:-}

Table (1) showed demographic criteria recorded regarding all our patients as Age, gender, BMI, biopsy class, hypertension, positive ANA, positive Anti ds-DNA, reduced C3 - C4 level, SLE duration in months, basal serum creatinine, 24 hours urine protein, e-GFR and serum albumin.

It showed that class IV (LN) is the most common class among all our patients and nearly $51.6 \%$ of our patients had hypertension.

There was no significant difference among the different classes of (LN) regarding urine volume/day, ESR, CRP, Hemoglobin level. Serum creatinine, liver enzyme, TSH, serum bilirubin, SLE duration, SLEDAI-2k and FBS, But there was a significant difference among different groups regarding 24 hrs Urinary protein on admission being the highest in class (V-III/IV) (LN) and a significant difference regarding serum albumin being the lowest in class (VIII/IV) (LN) as illustrated in table (2).

Comparison between the number of patients in each group - of the 3 classes of (LN) - who received induction by either oral MMF or iv Cyclophosphamide showed that the highest number of patients receiving iv CYC or oral MMF being in class IV (LN), however this did not show any statistically significant difference. As showed in Table (3).

The mucocutaneous, musculoskeletal, hematological and constitutional manifestations represented the highest percentage of manifestations up on which patients sought medical advice and were diagnosed as SLE patients. As illustrated in figure (1) 
We found that proteinuria, hypertensive emergency and nephritis flare were the 3 most common causes of admission of SLE cases in our unit and its percent is shown in figure (2).

We found that $31.3 \%$ of patients achieved CR, $45.3 \%$ achieved PR and $20.3 \%$ were non responders. As regards $\mathrm{CR}$, it was significantly different among the 3 groups being the highest in class III (LN) reaching 47.6\%, While PR was much noticed in class V-III/IV (LN), however it failed to establish a significant difference among other groups. Failure of treatment did not show any statistically significant difference among the 3 groups of patients table (4).

Table (5) shows that $9.4 \%$ of patient developed complications caused by treatment, while $10.9 \%$ developed ESRD and $17.2 \%$ died during the study - ( 2 of our patients died by unknown causes 3 weeks after starting treatment) -. However none of the 3 parameters of poor outcome showed a significant difference among the 3 classes of (LN).

There was no significant difference between the use of oral MMF or iv Cyclophosphamide, regarding CR or PR, However regarding non responders, a statistically significant difference was noticed as the number of non responders was higher in the CYC arm than the MMF arm reaching $30 \%$ of patients in the CYC group, facing only $4.2 \%$ in the MMF arm which can be applied as a point of superiority of MMF over iv CYC, Table (6) and table (7).

Table (8) shows rates of response between males and females with no significant difference; however despite being non significant our male patients showed a higher response rate either CR or PR than females.

Table (9) shows the difference between responders and non responders regarding SLEADI-2K showing a significant difference being higher in the non responders group.

While Table (10) shows a comparison between responders and non responders regarding demographic, clinical and investigational features. Base line e-GFR showed a significant difference among the 3 groups of responders being the lowest in the non responders \& the highest in CR. The higher the e-GFR, the better the response. The same did SLE duration in months which showed significant difference among patients being the lowest in CR \& The highest in the non responders. The longer SLE duration is associated with a poorer outcome.

Table 1:- Demographic characteristics of all our patients:

\begin{tabular}{|c|c|}
\hline $\begin{array}{l}\text { Gender: No. }(\%) \\
\text { Male } \\
\text { Female }\end{array}$ & $\begin{array}{l}7(10.9) \\
57(89.1)\end{array}$ \\
\hline $\begin{array}{l}\text { Biopsy class }(\mathrm{LN}): \text { No. }(\%) \\
\text { III } \\
\text { IV } \\
\text { V-III/IV }\end{array}$ & $\begin{array}{l}21(32.8) \\
28(43.8) \\
15(23.4)\end{array}$ \\
\hline $\begin{array}{l}\text { Hypertension: No. (\%) } \\
\text { With } \\
\text { Without }\end{array}$ & $\begin{array}{l}33(51.6) \\
31(48.4)\end{array}$ \\
\hline + ANA: No. (\%) & 64(100) \\
\hline + Anti ds-DNA: No. (\%) & $58(90.6)$ \\
\hline Reduced C3 \& C4 level: No. (\%) & 55(85.9) \\
\hline Age $(M \pm S D)$ & $27.4 \pm 7.3$ \\
\hline BMI $(M \pm \mathrm{SD})$ & $28.5 \pm 1.9$ \\
\hline SLE duration in months $(\mathrm{M} \pm \mathrm{SD})$ & $21.8 \pm 9.1$ \\
\hline Basal S.Cr.(mg/dl) $(\mathrm{M} \pm \mathrm{SD})$ & $3.6 \pm 2.2$ \\
\hline Basal e-GFR(ml/min/1.73m $\left.{ }^{2}\right)(M \pm S D)$ & $24.7 \pm 15.8$ \\
\hline Basal 24 hrs Urinary protein (gm/day) $(M \pm$ SD) & $4.4 \pm 2.3$ \\
\hline Albumin (g/dl) $(M \pm S D)$ & $2.6 \pm 0.5$ \\
\hline
\end{tabular}


Table 2:- Comparison between the three groups regarding routine laboratory data.

\begin{tabular}{|c|c|c|c|c|}
\hline & $\begin{array}{c}\text { III } \\
(\mathbf{A} / \mathrm{C})\end{array}$ & $\begin{array}{c}\text { IV } \\
(\mathrm{A} / \mathrm{C})\end{array}$ & $\begin{array}{c}\text { V-III/IV } \\
(\mathrm{A} / \mathrm{C})\end{array}$ & $\mathbf{P}$ \\
\hline Urine volume 24 hours & $\mathbf{9 7 3 . 8} \pm \mathbf{5 0 3 . 1}$ & $1212.5 \pm 589.5$ & $903.3 \pm 383.8$ & .123 \\
\hline ESR ( 1 st hour) & $98.4 \pm 25.2$ & $107.7 \pm 25.6$ & $105.5 \pm 13.5$ & .378 \\
\hline CRP & $1.9 \pm 0.8$ & $2.3 \pm 1$ & $2.3 \pm 1$ & .393 \\
\hline Hemoglobin g/dl & $9.5 \pm 0.8$ & $9.4 \pm 1.1$ & $9.5 \pm 1.1$ & .902 \\
\hline 24 hrs Urinary protein on admission g/day & $3.2 \pm 0.8$ & $3.6 \pm 1.1$ & $7.6 \pm 2.6$ & $<0.0011^{\hat{x}}$ \\
\hline S.Cr.(mg/dl) & $3.4 \pm 1.8$ & $3.8 \pm 2.5$ & $3.3 \pm 2$ & .718 \\
\hline + Anti ds-DNA: No. (\%) & 19(90.1) & $25(89.2)$ & 14(93.3) & .523 \\
\hline Reduced C3 \& C4 level: No. (\%) & 18(85.7) & $24(85.7)$ & 13(86.6) & .189 \\
\hline Serum Bilirubin $\quad$ T.(mg/dl) & $0.9 \pm 0.2$ & $0.6 \pm 0.7$ & $0.9 \pm 0.2$ & .140 \\
\hline Serum Bilirubin $\quad$ D.(mg/dl) & $0.1 \pm 0.1$ & $0.2 \pm 0.3$ & $0.1 \pm 0.1$ & .088 \\
\hline Albumin.( g/dl) & $2.8 \pm 0.5$ & $2.7 \pm 0.5$ & $2.2 \pm 0.6$ & .002 \\
\hline ALT (IU/I) & $21.2 \pm 7.2$ & $22.8 \pm 9.8$ & $19.4 \pm 3.7$ & .408 \\
\hline AST(IU/I) & $23.4 \pm 5.4$ & $24 \pm 9.9$ & $22.7 \pm 6$ & .866 \\
\hline INR & $1.1 \pm 0.3$ & $1.1 \pm 0.1$ & $1.2 \pm 0.1$ & .558 \\
\hline FBS(mg/dl) & $86.6 \pm 21.6$ & $91.6 \pm 11.3$ & $91.7 \pm 8.8$ & .463 \\
\hline TSH & $2.8 \pm 1.4$ & $2.7 \pm 1.2$ & $2.6 \pm 1.3$ & .862 \\
\hline Duration of SLE / months & $19.8 \pm 6.8$ & $23.8 \pm 9.6$ & $20.9 \pm 10.6$ & .285 \\
\hline SLEDAI-2K & $13.4 \pm 6.2$ & $16.1 \pm 7.1$ & $16.4 \pm 7.7$ & .317 \\
\hline
\end{tabular}

Table 3:- Correlation between the numbers of patients in each group receiving induction by the one of the 2 drugs:

\begin{tabular}{|c|c|c|c|c|c|c|}
\hline & \multicolumn{3}{|c|}{ Class } & \multirow[t]{2}{*}{ Total } & \multirow[t]{2}{*}{ p } \\
\hline & & III (A/C) & IV (A/C) & $\begin{array}{l}\text { V-III/IV } \\
\text { (A/C) }\end{array}$ & & \\
\hline Induction by MMF & No. $(\%)$ & 9(42.9) & $10(35.7)$ & $5(33.3)$ & $24(37.5)$ & 0.816 \\
\hline Induction by CYC & No. $(\%)$ & $12(57.1)$ & $18(64.3)$ & $10(66.7)$ & $40(62.5)$ & 0.411 \\
\hline
\end{tabular}

Figure 1:- The prevalence of different diagnostic criteria upon which patients were diagnosed as having SLE

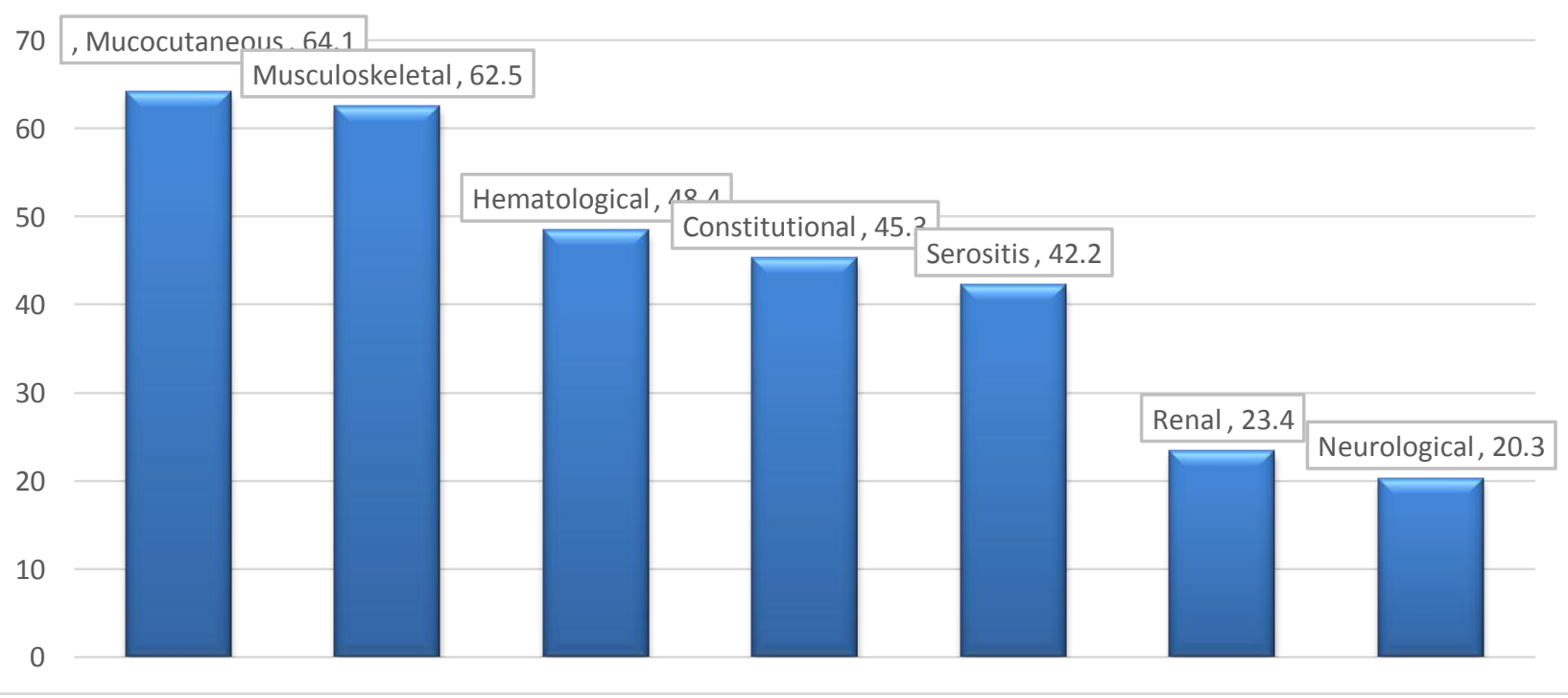


Figure 2:- The causes of admission of SLE patients at our nephrology unit

\section{Cause of admission}

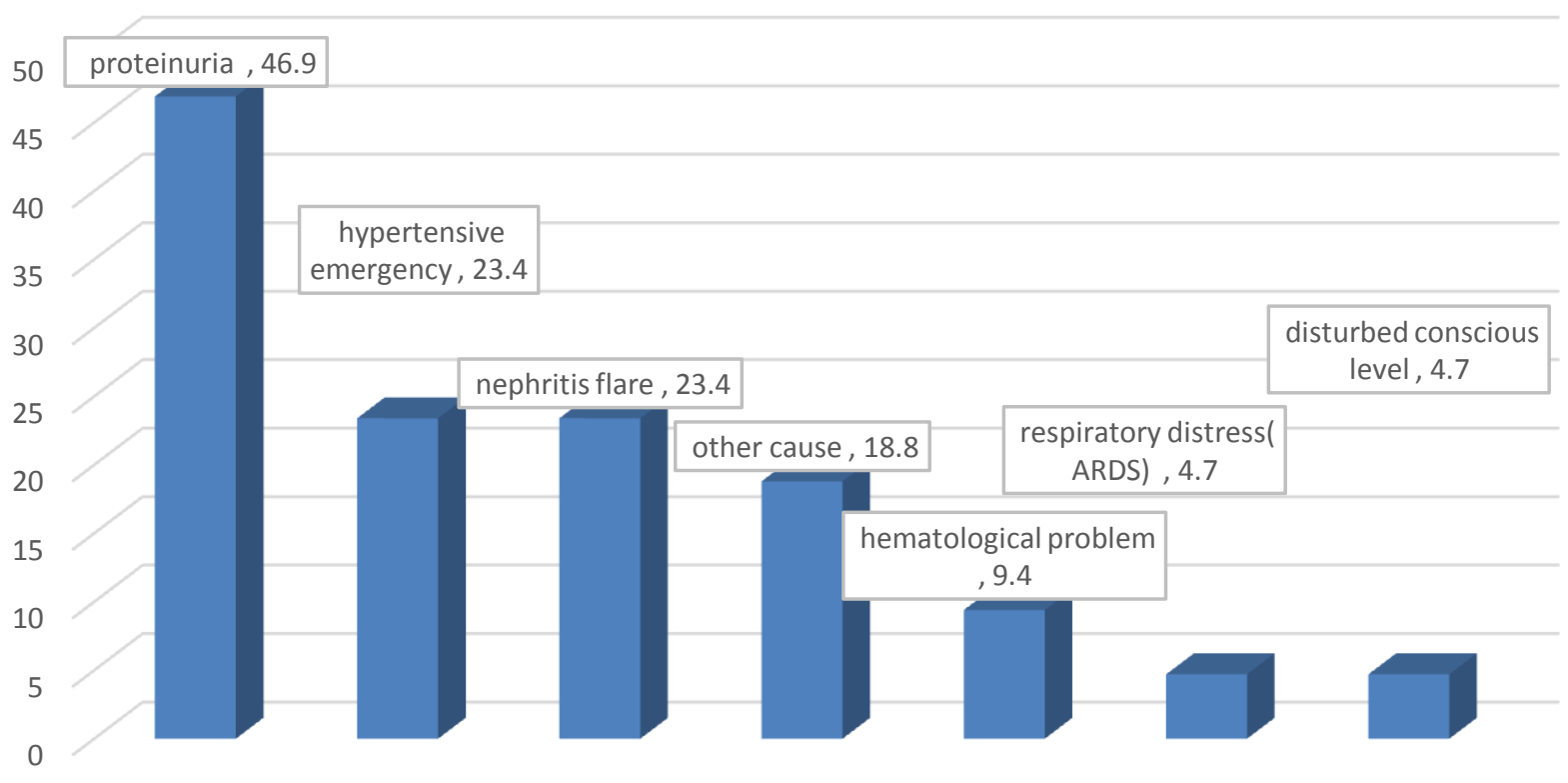

Table 4:- Comparison of rates of response among the 3 groups of patients

\begin{tabular}{|c|c|c|c|c|c|c|}
\hline & \multicolumn{3}{|c|}{ Class } & \multirow[t]{2}{*}{ Total } & \multirow[t]{2}{*}{$\mathbf{P}$} \\
\hline & & $\begin{array}{l}\text { III } \\
\text { (A/C) }\end{array}$ & $\begin{array}{l}\text { IV } \\
(\mathrm{A} / \mathrm{C})\end{array}$ & $\begin{array}{l}\text { V-III/IV } \\
\text { (A/C) }\end{array}$ & & \\
\hline $\begin{array}{l}\text { complete } \\
\text { Remission }\end{array}$ & No $(\%)$ & $10(47.6)$ & $9(32.1)$ & $1(6.7)$ & $20(31.3)$ & 0.033* \\
\hline Partial remission & No $(\%)$ & $7(33.3)$ & 12(42.9) & $10(66.7)$ & 29(45.3) & 0.132 \\
\hline $\begin{array}{l}\text { Non- } \\
\text { Responders }\end{array}$ & No (\%) & 4(19) & $6(21.4)$ & $3(20)$ & $13(20.3)$ & 0.979 \\
\hline
\end{tabular}

Table 5:- Comparison of rates of poor outcome among the 3 groups of patients

\begin{tabular}{|c|c|c|c|c|c|c|}
\hline & \multicolumn{3}{|c|}{ Class } & \multirow[t]{2}{*}{ Total } & \multirow[t]{2}{*}{$\mathbf{P}$} \\
\hline & & $\begin{array}{l}\text { III } \\
(\mathrm{A} / \mathrm{C})\end{array}$ & $\begin{array}{l}\text { IV } \\
(\mathrm{A} / \mathrm{C})\end{array}$ & $\begin{array}{l}\text { V-III/IV } \\
\text { (A/C) }\end{array}$ & & \\
\hline $\begin{array}{l}\text { Treatment } \\
\text { complications }\end{array}$ & No.(\%) & $\mathbf{0}(\mathbf{0})$ & $4(14.3)$ & $2(13.3)$ & $6(9.4)$ & 0.198 \\
\hline ESRD & No. $(\%)$ & $3(14.3)$ & $4(14.3)$ & $\mathbf{0}(\mathbf{0})$ & $7(10.9)$ & 0.3 \\
\hline Death & No. $(\%)$ & $2(9.5)$ & $6(21.4)$ & $3(20)$ & $11(17.2)$ & 0.521 \\
\hline
\end{tabular}

Table 6:- comparison of rates of response between the 2 arms of induction

\begin{tabular}{|c|c|c|c|c|c|}
\hline & \multicolumn{2}{|c|}{ Induction Regimen } & \multirow[t]{2}{*}{ Total } & \multirow[t]{2}{*}{$\mathbf{P}$} \\
\hline & & MMF & CYC & & \\
\hline Complete Remission & No.(\%) & $9(37.5)$ & 11(27.5) & $20(31.3)$ & .403 \\
\hline Partial remission & No.(\%) & 12(50) & $17(42.5)$ & $29(45.3)$ & .560 \\
\hline Non- Responders & No.(\%) & 1(4.2) & $12(30)$ & $13(20.3)$ & $.013^{x}$ \\
\hline
\end{tabular}


Table 7:- comparison between the 2 arms of induction regarding poor outcome.

\begin{tabular}{|c|c|c|c|c|c|}
\hline & \multicolumn{2}{|c|}{ Induction Regimen } & \multirow[t]{2}{*}{ Total } & \multirow[t]{2}{*}{$\bar{P}$} \\
\hline & & MMF & CYC & & \\
\hline $\begin{array}{l}\text { Treatment } \\
\text { complications }\end{array}$ & No.(\%) & $1(4.2)$ & $5(12.5)$ & $6(9.4)$ & .268 \\
\hline ESRD & No.(\%) & 1(4.2) & $6(15)$ & $7(10.9)$ & .179 \\
\hline Death & No.(\%) & $3(12.5)$ & $8(20)$ & 11(17.2) & .441 \\
\hline
\end{tabular}

Table 8:- comparison between Male and female response

\begin{tabular}{|l|l|l|l|l|l|l|}
\hline \multicolumn{2}{|c|}{} & \multicolumn{3}{|c|}{ Pesponse } \\
\cline { 3 - 6 } \multirow{2}{*}{ Gender } & Female & No.(\%) & CR & PR & NR & \\
\cline { 2 - 5 } & Male & No.(\%) & $\mathbf{2 ( 1 0 )}$ & $\mathbf{2 5}(\mathbf{8 6 . 2})$ & $\mathbf{1 2 ( 9 2 . 3 )}$ & \\
& &
\end{tabular}

Table 9:- SLEADI-2k between responders and non responders

\begin{tabular}{|l|c|c|c|}
\hline & \multicolumn{1}{|c|}{$\begin{array}{c}\text { Responders } \\
(49)\end{array}$} & \multicolumn{1}{c|}{$\begin{array}{c}\text { Non- Responders } \\
(13)\end{array}$} & P \\
\hline SLEADI-2K & $12.9 \pm 6$ & $\mathbf{2 4 . 6 \pm 6}$ & $<0.001^{\times}$ \\
\hline
\end{tabular}

Table 10:- Comparison between different factors and treatment response

\begin{tabular}{|c|c|c|c|c|}
\hline & $\begin{array}{c}\mathrm{CR} \\
\mathrm{N}=20\end{array}$ & $\begin{array}{c}\text { PR } \\
\mathbf{N}=29\end{array}$ & $\begin{array}{c}\mathrm{NR} \\
\mathrm{N}=13\end{array}$ & $\mathbf{P}$ \\
\hline Age & $27 \pm 7.2$ & $28.5 \pm 7.6$ & $25.6 \pm 7$ & .478 \\
\hline BMI & $28.1 \pm 1.9$ & $28.4 \pm 2$ & $29.4 \pm 1.6$ & .163 \\
\hline $\mathbf{H b}$ & $9.2 \pm 0.8$ & $9.8 \pm 1.1$ & $9.2 \pm 1$ & .078 \\
\hline $\begin{array}{l}\text { Baseline } 24 \text { hrs Urinary Protein } \\
\text { (gm/day) }\end{array}$ & $3.7 \pm 1.2$ & $4.8 \pm 2.5$ & $4.7 \pm 3$ & .199 \\
\hline Baseline e-GFR & $28.8 \pm 15$ & $28.2 \pm 16.1$ & $11.3 \pm 7.5$ & $.001^{\mathrm{x}}$ \\
\hline Baseline S.Cr (mg/dl) & $3.8 \pm 2.3$ & $3.4 \pm 2.1$ & $3.5 \pm 2.1$ & .808 \\
\hline Urine volume/ 24 hours & $1047.6 \pm 492.1$ & $1101.7 \pm 546.8$ & $1000 \pm 579.5$ & .835 \\
\hline SLE duration in months & $17.2 \pm 6.8$ & $23.4 \pm 9.7$ & $25.3 \pm 8.7$ & .014 \\
\hline
\end{tabular}

\section{Discussion:}

Systemic Lupus Erythematosus (SLE) is a multisystem autoimmune disorder with a broad spectrum of clinical presentations. It can virtually involve any self structure of the body and exhibits a large spectrum of clinical manifestations including cutaneous and joint diseases, renal diseases, hematological involvements and central nervous system diseases Lee et al., (2010) (2).

Lupus nephritis (LN) occurs in up to $60 \%$ of adults with SLE and predicts poor survival. The prevalence of SLE and (LN) and treatment response vary by age, gender, location and race/ethnicity Jakes et al., (2012) (1).

Lupus-associated renal damage has been one of the leading causes of 5-year mortality in patients with SLE in the past 50 years. (LN) also entails substantial productivity loss, reduction in health-related quality of life and a psychosocial burden in patients with SLE Mak et al., (2012). (28).

In this study the predominance of female sex was similar to all western studies (Hsu et al., 2011) (27). But regarding the ratio of female to male it was (8.1:1) which came in consistent with the finding of Al Arfaj and Khalil., (2009) (18) in KSA who reported the same ratio and was close to the finding of a study carried out in India by Sircar et al., (2013) (20) but higher than reported by Pakozdi et al.,( 2016) (12) in a study carried out in India who reported a ratio of female to male (4.7: 1)\& and higher than reported by Yi et al., (2015) (8) in a study carried out in China who reported a ratio of female to male (5.7: 1), the difference in this values can be attributed to Ethnic \& genetic differences.

In this study the Mean \pm SD duration of SLE in months was $21.8 \pm 9.1$, that was nearly similar to the study by Sircar et al., (2013) (20) in India in which the Mean \pm SD disease duration in months was 13.9 \pm 4.1 months, but 
was totally far than the study by Galindo-Izquierdo et al., (2016) (6) in Spain in which the Mean disease duration in months was 124.4 months, this major difference can be attributed to that in the Spanish study a very huge sample size was chosen for the study reaching 3350 patients of whom 1092 developed lupus nephritis .

However, In our study it was a single centre study in comparison to the Spanish study, but still supported by Hanly et al., (2016) (26) who stated that "Whereas the screening frequency depends on disease severity and prior history of (LN), we are especially vigilant in the first 6 months of SLE diagnosis when new (LN) most often occurs".

In this study all our patients had proteinuria , $4.4 \pm 2.3 \mathrm{gm} /$ day which necessitate renal biopsy revealing (LN) of the following classes ( III , IV and V/III or V/IV) according to ISN/RPS classification, this was consistent with Yi et al., (2015) (8) study in which proteinuria level was $5.64 \pm 4.65 \mathrm{~g} /$ day, but higher than Galindo-Izquierdo et al., (2016) (6) in which proteinuria level was $2.5(1.2-4.4) \mathrm{g} / \mathrm{day}$. This high level of proteinuria can be attributed to that most patients - with SLE - had presented to us for the new appearance of proteinuria ( $46.9 \%$ ) or the occurrence of (LN) flares (23.4\%) which was unfortunately cannot be judged for the absence of a previous renal biopsy or enough data about the patients. These 2 causes can contribute to the high level of proteinuria in our patients.

In this study (LN) occurred in almost 100\% of patients, this was supported by Yap et al., (2012) (25) \& Yap and Chan., (2015) (24) but on the contrary of Galindo-Izquierdo et al., 2016 (6) in which (LN) represented 30.5\% of patients consistent with the frequency reported in the literature, which ranges between 25 and $60 \%$. This controversy can be attributed to that a large number of our patients was admitted for developing proteinuria or the presence of a lupus nephritis flare besides Ishizaki et al., 2015 (23) stated that the actual prevalence of (LN) is inferred to be higher than reported as class III/IV (LN)- presenting with proliferative GN and associated with poor prognosis accounts for $15-20 \%$ of cases of silent (LN).

In our study, class IV (LN) represented $43.8 \%$ (28/64) of all our patients, with class III (LN) represented 32.8\% (21/64) and class V-III/IV (LN) represented 23.4\% (15/64), As shown above, (LN) class IV is the commonest \& this is consistent with Galindo-Izquierdo et al., (2016) (6) in which (LN) class IV was 48.7\% (433/1092) but lower than Pakozdi et al., (2016) (12) in which Class III (LN) 36\% (31/86) and Class IV (LN) (64\%)(55/86); and much lower than Sircar et al., (2013) (20) in which (LN) class IV was $82.5 \%$ ( 71/86).

In this study, other histological features in biopsies other than those illustrated by the International Society of Nephrology/Renal Pathology Society ISN/RPS were put aside, This was supported by Hsieh et al., (2011) (22), Hahn et al., (2012) (15), Moroni et al., (2016) (13) \& Wilhelmus et al., (2016) (21) who stated that the significance of other biopsy findings, in affecting treatment responsiveness and outcomes, is not yet well characterized .

Regarding comparative levels of proteinuria \& serum albumin between different classes of (LN), proteinuria was higher in the mixed Class ( V+ III/IV) than other 2 groups reaching $7.6 \pm 2.6 \mathrm{~g} /$ day \& serum albumin was reduced than other 2 groups reaching $2.2 \pm 0.6 \mathrm{~g} / \mathrm{dl}$, this comes in consistency with Ikeuchi et al., (2016) (14) who reported proteinuria of 5.0 (2.2-6.6) g/day which was higher than pure Class III/IV and a serum albumin of $2.4 \pm 0.7 \mathrm{~g} / \mathrm{dl}$ which was lower than pure Class III/IV.

In this study, the rates of response have been $31.3 \%$ (20/64) for complete remission \& $45.30 \%$ (29/64) for partial remission, these results are higher than those for Sircar et al., (2013) (20) in which the rates of response, have been $21 \%$ and $23 \%$ for CR and PR respectively, lower than Yi et al., (2015) (8) in which the rates of response, have been 52\% and $15.7 \%$ for CR and PR respectively \& much lower than Galindo-Izquierdo et al., 2016 (6) in which CR has been $68.3 \%$.

In this study, $20.3 \%$ of patients reported treatment failure which is higher than Sircar et al., (2013) (20) who reported that $14 \%$ of the patients experienced treatment failure after the first 6 months. This is a little bit higher than the rate of treatment failure in the Euro Lupus trial (16\% in the low-dose cyclophosphamide arm; $20 \%$ in the high-dose arm), This could be due to the fact that most of our patients belonged to a high risk category with low socio-economic status Houssiau et al., (2002) (19).

In this study , $10.9 \%$ developed ESRD (7/64) while 17.2\% died (11/64) which was near to Al Arfaj and Khalil., (2009) (18) in KSA who reported 9\% developed ESRD \& 6\% for death, lower than results reported by Singh et al., 
(2011) (17) in USA who reported (29.5\%) patients progressed to ESRD (36/122) \& similar to results reported by Yi et al., (2015) (8) who reported 13.9\% developed ESRD \& $5.3 \%$ for death\& Galindo-Izquierdo et al., (2016) (6) who reported $10.35 \%$ for ESRD. ESRD was defined the same as Galindo-Izquierdo et al., (2016) (6) by the need of dialysis and/or transplantation.

The high rate of death in our study can be attributed to complications of treatment as infections \& ARDS (5/11). It should be mentioned that lupus nephritis itself has a poor prognostic risk factor of mortality Galindo-Izquierdo et al., (2016) (6).

Among those who developed ESRD (7/64) 4 patients had (LN) class IV, this correlates with Tektonidou et al., 2016 (16) who stated that patients with class IV (LN) are especially vulnerable to ESRD. In this study the highest rate of treatment complications specially infection was associated with the use of Cyclophsphamide induction (6/64) ( 5 of the 6 patient on CYC), that is why the induction course of cyclophosphamide should be limited to no more than 6 months Hahn et al., (2012) (15).

In this study, among those who showed treatment response either CR or PR, the highest group was that with class III (LN) reaching $80.9 \%$ (17/21) (47.6\% for CR \& 33.3\% for PR), While among the non responders the highest was in class IV (LN) \& class (V-III/IV) reaching $21.4 \%(6 / 28) \& 20 \%(3 / 15)$ respectively. The poor outcome in this study lies within class IV \& class (V-III/IV). Our results were similar to Yi et al., (2015) (8) who noticed much severer organ damage in class IV (LN) patients but these results were faced by Ikeuchi et al., (2016) (14) who stated that Class $(\mathrm{V}+\mathrm{III} / \mathrm{IV})(\mathrm{LN})$ had significantly poorer renal outcomes than patients with pure Class III/IV $(\mathrm{LN})$.

Ikeuchi et al., (2016) (14) stated that It is worth to be mentioned that," the effect of Class V lesions with concurrent Class III or IV lesions on renal survival remains unclear. Till recently, in many reports using the ISN/ RPS 2003 classification, proliferative (LN) (Class III or Class IV) combined with membranous lesions was classified as Class III or Class IV, respectively. Old series reported that membranous nephropathy had the worst prognosis among other histological classes of (LN), particularly when signs of proliferation or inflammation are present, while other reports pointed out not only a good long-term renal survival in lupus patients with pure membranous nephropathy but also the lack of significant difference between pure forms and mixed forms. Moroni et al., (2016) (13).

Regarding the line of treatment used for induction, In this study despite the presence of a higher response rate either regarding CR or PR in MMF group more than Cyclophosphamide (CYC) group ( CR was 37.5\% \& $27.5 \%$ in MMF \& CYC respectively \& PR was 50\% \& 42.5\% in MMF \& CYC respectively ), but was not statistically significantly different. However, there was a statistically significant difference between the 2 arms regarding the non responders, being higher in the CYC arm, which suggests an element of superiority of MMF over CYC, this is supported by Pakozdi et al., (2016) (12) who reported that MMF offers equivalent or in some studies higher remission rates than IV CYC for (LN) induction treatment Ginzler et al., (2005) and Rathi et al., (2016) (10-11).

In this study, we could not find a statistically significant difference in response between males \& females. In fact, the response rate (either PR or CR ) was surprisingly higher in males than in females reaching $\{85.7 \%(6 / 7)$ in males \& 75.4\% (43/57) in females \}, although this can be supported by Laura et al., (2016) (9) who stated that" Male sex was not associated with ESRD incidence", this is not in concordance with other studies as Yi et al., (2015) (8) who stated that "the disease severities and organs damage (especially renal impairment) of (LN) in male patients are higher than those of female patients, which was consistent with other studies". This difference can be attributed to the small sample size in our study.

In this study patients with high SLEDAI-2K score developed poorer outcome than those with low score, this correlates with Yi et al., (2015) (8) who reported that $30.9 \%$ patients with SLEDAI score > 20 developed ESRD during follow-up.

In this study the response was influenced by SLE duration in months significantly. The shorter the duration the better the response. For patients who achieved CR, the SLE duration in months mean was 17.2 \pm 6.8 , while those who were considered non responders the SLE duration in months mean was $25.3 \pm 8.7$.

These results are similar to the previously reported, suggesting that delayed (LN) development tends to progress in tandem with renal damage compared to the good therapeutic response that (LN) typically manifests at SLE onset 
Takahashi et al., (2009) (7). However, this come in contrast with The Spanish study in which the onset time of (LN) during the course of SLE did not affect the outcome of renal function Galindo-Izquierdo et al., (2016) (6).

\section{Conclusion:-}

In conclusion, the results of our study revealed the high prevalence of class IV (LN) among SLE patients, the little superiority of MMF over cyclophosphamide in induction of remission and the better outcome of class III lupus nephritis over other classes.

Given the poor outcome of (LN) among SLE patients, depending on the class of the biopsy, our findings suggest that future studies are warranted with special stress on the mixed class of (V-III/IV) (LN) with the consensus on international definitions of remission and treatment failure beside the introduction of new drugs and local guidelines based on clinical trials.

\section{References:-}

1. Jakes RW, Bae SC, Louthrenoo W., et al., (2012). Systematic review of the epidemiology of systemic lupus erythematosus in the Asia- Pacific region: prevalence, incidence, clinical features, and mortality. Arthritis Care Res (Hoboken) .64(2):159-168.

2. Lee $\mathbf{Y}$, Woo JH, Choi S, et al.. (2010). Induction and maintenance therapy for lupus nephritis: a systematic review and meta-analysis. Lupus. 19: 703-710.

3. Hoover PJ and Costenbader KH., (2016). Insights into the epidemiology and management of lupus nephritis from the US rheumatologist's perspective. Kidney Int. 90(3):487-92.

4. D'Cruz D and Houssiau F, (2009). The Euro-Lupus Nephritis Trial: the development of the sequential treatment protocol. Lupus; 18: 875-877

5. Kalloo S, Aggarwal N, Mohan P., et al., (2013). Lupus nephritis: treatment of resistant disease. Clin J Am Soc Nephrol.;8:154-61.

6. Galindo-Izquierdo M, Rodriguez-Almaraz E, Pego-Reigosa JM., et al., (2016). RELESSER Group, from Spanish Society of Rheumatology Systemic Autoimmune Diseases Study Group (EASSER). Characterization of Patients With Lupus Nephritis Included in a Large Cohort From the Spanish Society of Rheumatology Registry of Patients With Systemic Lupus Erythematosus (RELESSER) Medicine (Baltimore).95(9):e289.

7. Takahashi Y, Mizoue T, Suzuki A., et al., (2009). Time of initial appearance of renal symptoms in the course of systemic lupus erythematosus as a prognostic factor for lupus nephritis. Mod Rheumatol. 19:293-301.

8. Yi Tang, XiaoYan Zhang, Ling Ji., et al., (2015). Clinicopathological and outcome analysis of adult lupus nephritis patients in China. Int Urol Nephrol. 47:513-520.

9. Laura Plantinga, S. Sam Lim, Rachel Patzer., et al., (2016). Incidence of End-Stage Renal Disease Among Newly Diagnosed Systemic Lupus Erythematosus Patients: The Georgia Lupus Registry. Arthritis Care \& Research . 68(3) : 357-365.

10. Ginzler EM, Dooley MA, Aranow C., et al., (2005). Mycophenolate mofetil or intravenous cyclophosphamide for lupus nephritis. N Engl J Med. 353:2219-28.

11. Rathi M, Goyal A, Jaryal A., et al., (2016). Comparison of low-dose intravenous cyclophosphamide with oral mycophenolate mofetil in the treatment of lupusnephritis. Kidney Int.89:235-42.

12. Pakozdi A, Rajakariar R, Sheaff M., et al., (2016). Treatment outcomes from a multiethnic lupus cohort with proliferative nephritis. Indian J Rheumatol . 11:14-21.

13. Moroni G, Depetri F and Ponticelli C., (2016). Lupus nephritis: When and how often to biopsy and what does it mean?, Journal of Autoimmunity.74: 27-40.

14. Ikeuchi $\mathbf{H}$, Hiromura $\mathbf{K}$, Kayakabe $\mathbf{K}$., et al., (2016). Renal outcomes in mixed proliferative and membranous lupus nephritis (Class III/IV + V): A long-term observational study. Mod Rheumatol.26:1-6.

15. Hahn BH, McMahon MA, Wilkinson A., et al., (2012). American College of Rheumatology. American College of Rheumatology guidelines for screening, treatment, and management of lupus nephritis. Arthritis Care Res (Hoboken). 64: 797-808.

16. Tektonidou MG, Dasgupta A and Ward MM., (2016). Risk of end-stage renal disease in patients with lupus nephritis, 1970 to 2015: a systematic review and Bayesian meta-analysis. Arthritis Rheumatol. 68(6):1432-41.

17. Singh Sandeep, Xin J. Zhou, Chul Ahn., et al., (2011). .Lupus Nephritis. A Retrospective Analysis of Clinical Presentation and Outcomes from a single center Am J Med Sci. 342(6): 467-473. 
18. Al Arfaj AS and Khalil N., (2009). Clinical and immunological manifestations in 624 SLE patients in Saudi Arabia. Lupus.18:465-73.

19. Houssiau FA, Vasconcelos C, D’Cruz D., et al., (2002). Immunosuppressive therapy in lupus nephritis: The euro-lupus nephritis trial, a randomized trial of low-dose versus high-dose intravenous cyclophosphamide. Arthritis Rheum.46:2121-31

20. Sircar D , Sircar G, Waikhom R., et al., (2013). Clinical features, epidemiology, and short-term outcomes of proliferative lupus nephritis in Eastern India. Indian Journal of Nephrology . 23(1):5-11.

21. Wilhelmus S, Bajema IM, Bertsias GK., et al., (2016). Lupus nephritis management guidelines compared . Nephrol Dial Transplant. 31(6):904-13

22. Hsieh C, Chang A, Brandt D., et al., (2011). Predicting outcomes of lupus nephritis with tubulointerstitial inflammation and scarring. Arthritis Care Res (Hoboken). 63: 865-874.

23. Ishizaki J, Saito K, Nawata M, et al., (2015). Low complements and high titre of anti-Sm antibody as predictors of histopathologically proven silent lupus nephritis without abnormal urinalysis in patients with systemic lupus erythematosus. Rheumatology (Oxford). 54(3):405-12.

24. Yap DY and Chan TM., (2015). Lupus Nephritis in Asia: Clinical Features and Management. Kidney Dis. 1(2):100-9.

25. Yap DY, Tang CS, Ma MK., et al., (2012). Survival analysis and causes of mortality in patients with lupus nephritis. Nephrol Dial Transplant.27:3248-3254.

26. Hanly JG, O'Keeffe AG, Su L., et al., (2016). The frequency and outcome of lupus nephritis: results from an international inception cohort study. Rheumatology (Oxford). 55:252-262.

27. Hsu CY, Chiu WC, Yang TS., et al., (2011). Age- and gender-related long-term renal outcome in patients with lupus nephritis. Lupus. 20(11):1135-1141.

28. Mak A, Cheung MW, Chiew HJ., et al., (2012). Global trend of survival and damage of systemic lupus erythematosus: meta-analysis and meta-regression of observational studies from the 1950s to 2000s. Semin Arthritis Rheum. 41:830-9.

29. KDIGO., (2012). KDIGO Clinical Practice Guideline for Glomerulonephritis. Kidney Int Suppl. 2:209.

30. Ruiz-Irastorza G, Ramos-Casals M, Brito-Zeron P, et al., (2010). Clinical efficacy and side effects of antimalarials in systemic lupus erythematosus: a systematic review. Ann Rheum Dis . 69: 20-28. 